\title{
Lynch syndrome-chasing a better ascertainment rate in British Columbia
}

\author{
Carol Cremin*, Morteza Bashash, Linlea Armstrong, Sharlene Gill, David Huntsman, Chris Bajdik \\ From 13th Annual Meeting of the Collaborative Group of the Americas on Inherited Colorectal Cancer \\ Honolulu, Hawaii, USA. 16-17 October 2009
}

\section{Background}

With a population of approximately 4.380 million people and an estimated Lynch syndrome mutation prevalence of 1/531, there are an expected 8000 individuals with Lynch syndrome in British Columbia. The Hereditary Cancer Program (HCP) of the BC Cancer Agency (BCCA) has provided clinical testing for Lynch syndrome since 2004 to patients across the province. Currently, there are approximately 100 patients with confirmed Lynch syndrome mutations in the BCCA database. Potential obstacles in ascertaining Lynch syndrome through a traditional clinic-based approach include physician awareness of referral criteria, patient's lack of knowledge of cancer family history, patient compliance, and availability of tumour tissue for testing. Given these obstacles, a population based approach to identifying Lynch syndrome through incident testing of newly diagnosed colorectal cancers under age 50 by microsatellite instability testing (MSI) was launched in BC in June of 2008.

\section{Methods}

Chart review of a cohort of patients referred for genetic counselling at the RCP during 2004-2006 and a cohort of consecutive colorectal cancer cases referred directly for MSI testing to the BCCA Genetics Laboratory (June 2008-June 2009). Mutation prevalence and clinicopathologic characteristics will be compared between the two groups. Clinical and demographic characteristics of the groups will also be compared to non-referred cases diagnosed under 50 in the province.

\section{Results}

Our previous clinic-based results showed a $14.3 \%$ prevalence of Lynch syndrome mutations among the index cases tested for whom results were available. $76 \%$ of tumour results were microsatellite stable and intact for MLH1 and MSH2 proteins. The sensitivity of the program's referral criteria was about $83.3 \%$ with an approximate confidence interval of $68.2 \%-96.8 \%$ and the positive predictive value was about $38.3 \%$ with an approximate confidence interval of $17.7 \%-60.0 \%$. The prevalence of Lynch syndrome mutations dropped to $3.2 \%$ among all patients referred for genetic counseling. From July 2008 to July 2009, a total of 37 incident colorectal cases diagnosed under age 50 were referred directly to the BC Cancer Agency's cancer genetics lab. $73 \%$ were microsatellite stable while additional testing is underway on the $10 \mathrm{MSI}$ high cases. Further comparisons between the groups will be presented.

\section{Conclusion}

Aside from becoming increasingly important for prognosis and predictive response to chemotherapy, population based MSI analysis on newly diagnosed colorectal cancer is expected to improve the rate of Lynch syndrome ascertainment in $\mathrm{BC}$.

Published: 25 May 2010

doi:10.1186/1897-4287-8-S1-P5

Cite this article as: Cremin et al:: Lynch syndrome-chasing a better ascertainment rate in British Columbia. Hereditary Cancer in Clinical

Practice 2010 8(Suppl 1):P5.

\footnotetext{
* Correspondence: ccremin@bccancer.bc.ca

BC Cancer Agency, Hereditary Cancer Program, Vancouver V5Z 1H5 British Columbia, Canada
} 